Biophysical Reviews and Letters

(C) World Scientific Publishing Company

\title{
Interacting single-file system: Fractional Langevin formulation versus diffusion-noise approach
}

\author{
ALESSANDRO TALONI \\ CNR-IENI, Via R. Cozzi 53 \\ Milano, 20125, Italy \\ alessandro.taloni@gmail.com \\ FABIO MARCHESONI \\ Dipartimento di Fisica, Universitá di Camerino, Via Madonna delle Carceri, 9 \\ Camerino, I-62032, Italy \\ fabio.marchesoni@pg.infn.it \\ Received Day Month Year \\ Revised Day Month Year
}

\begin{abstract}
We review the latest advances in the analytical modelling of single file diffusion. We focus first on the derivation of the fractional Langevin equation that describes the motion of a tagged file particle. We then propose an alternative derivation of the very same stochastic equation by starting from the diffusion-noise formalism for the time evolution of the file density.
\end{abstract}

Keywords: single file model; fractional Langevin equation; diffusion noise equation

\section{Introduction}

The single file model describes particle diffusion through channels or pores. Firstly introduced in the biological context? ?, single file diffusion (SFD) gained widespread interest in the mathematical physical community ???, ?, ?, ?, ?, as a simple, analytically tractable model demonstrating anomalous diffusion. Due to the simplicity of its formulation, the limited number of the parameters characterizing its dynamics, and its immediate relation to real physical phenomena, such a stylized model provides an ideal benchmark against which we can test our understanding of anomalous diffusion.

The single file model is defined as an assembly of identical interacting particles diffusing on a one dimensional (1D) substrate. The particles are non-passing, i.e. they retain their initial file ordering at any time, a spatial constraint which goes under the name of single filing condition. This means that one can pick any file particle and attempt to describe its stochastic dynamics; this particle is usually referred to as the tagged particle. It has been known since the very first formulation of the model ? , that the mean square displacement of the tagged particle undergoes 
the asymptotic law

$$
\left\langle\left[x_{n}(t)-x_{n}(0)\right]^{2}\right\rangle=2 \sqrt{\frac{D t}{\pi\langle\rho\rangle^{2}}},
$$

where $D$ is the diffusion coefficient of the freely diffusing particles and $\langle\rho\rangle$ denotes the average equilibrium file density.

In this paper we review some of the major advances in the analytical treatment of single file systems achieved over the last decade. First, we focus on the action of an external potential and how it affects the tagged particle dynamics. Second, we discuss two alternative descriptions of the SFD: one, called fractional Langevin approach, allows to extract an effective stochastic equation for the tagged particle from the detailed file dynamics. The other one, termed diffusion-noise approach, introduces an intermediate step, namely, the equation for time evolution of the file density; the fractional Langevin equation of the tagged particle is then derived by solving the relevant density equation.

\subsection{Interacting single file system}

Let us consider a $1 \mathrm{D}$ file of $N$ pointlike Brownian particles moving on a ring according to the Langevin equations

$$
\frac{d^{2} x_{n}(t)}{d t^{2}}=-\gamma \frac{d x_{n}(t)}{d t}-\frac{\partial}{\partial x_{n}} U\left(x_{1}, \ldots, x_{N} ; t\right)+\eta_{n}(t)
$$

with $n=1, \ldots, N$, where the damping constant $\gamma$ and the noises $\eta_{n}(t)$ satisfy the fluctuation-dissipation relations

$$
\left\langle\eta_{i}(t) \eta_{j}\left(t^{\prime}\right)\right\rangle=2 k_{B} T \gamma \delta_{i, j} \delta\left(t-t^{\prime}\right) .
$$

where $\delta$ stands for the Dirac's delta function. The file particles interact with both their nearest neighbors and the environment. The potential function in Eq. (2) consists of two contributions,

$$
U\left(x_{1}, \ldots, x_{N} ; t\right)=\sum_{n, m=1} U_{H C}\left(\left|x_{n}-x_{m}\right|\right)+U_{\text {int }}\left(x_{1}, \ldots, x_{N} ; t\right),
$$

with the hard-core repulsion

$$
U_{H C}\left(\left|x_{n}-x_{m}\right|\right)=\left\{\begin{array}{cc}
\infty & \left|x_{n}-x_{m}\right|=0 \\
0 & \text { otherwise }
\end{array}\right.
$$

establishing the filing condition. As stated at the section beginning in the following we will focus on the zero particles' size limit, however it must be stressed that the truly interaction potential for particles with a finite size $a$ yields $U_{H C}\left(\left|x_{n}-x_{m}\right|\right)=$ $\infty$ if $\left|x_{n}-x_{m}\right|=a$. The additional substrate potential $U_{\text {int }}$, is often introduced to model distinct physical situations. 
- File interaction with a substrate. A typical example is represented by nonpassing particles confined to constrained geometries, like compartmentalized narrow channels ? In this case the $U_{\text {int }}$ maxima model the entropic barriers opposing the particle diffusion through the compartment pores. The most general form of such interaction potential is given by

$$
U_{\text {int }}\left(x_{1}, \ldots, x_{N} ; t\right)=\int d X \sum_{n=1} U(X ; t) \delta\left(X-x_{n}\right) .
$$

Note that the spatial coordinate, $X$, has been capitalized so as to be distinguished from the particle trajectories, $x_{1}(t), \ldots, x_{N}(t)$. A substantial simplification comes from the assumption that the potential is timeindependent, i.e $U(X ; t) \equiv U(X)$. The first example of substrate po[tential energy reported in the literature,

$$
U(X)=d\left[1-\cos \left(\frac{2 \pi X}{l}\right)\right],
$$

modeled the periodic corrugations of a narrow quasi 1D channel ?. In Eq.(7) $d$ and $l$ denote respectively the amplitude and period of the channel wall corrugation. The authors of Refs. ?? established a general expression for the tagged particle diffusion in mirror symmetric potentials, which they were able to compute explicitly only for the harmonic potential

$$
U(X)=\frac{\omega^{2}}{2} X^{2} .
$$

Their results corroborated, both numerically and theoretically, the validity of the so-called Percus diffusion formula?

$$
\left\langle\left[x_{n}(t)-x_{n}(0)\right]^{2}\right\rangle=\frac{\left\langle\left|x_{\text {free }}(t)-x_{\text {free }}(0)\right|\right\rangle}{\langle\rho\rangle},
$$

where $x_{\text {free }}(t)$ represents the trajectory of a single (passing) particle under the conditions given. The Percus' rule holds whenever the mean square displacement is described in the restframe of the center of mass. In fact, Percus' formula applies to any kind of single particle dynamics, namely, to Brownian particles, like in Eq.(2), as well as to anomalous diffusing particles ???????. For instance, if the free diffusion law of a single particle is

$$
\left\langle\left[x_{\text {free }}(t)-x_{\text {free }}(0)\right]^{2}\right\rangle \propto t^{\alpha},
$$

with $\alpha>0$, then, from Eq.(9) one obtains $\left\langle\left[x_{n}(t)-x_{n}(0)\right]^{2}\right\rangle \propto t^{\alpha / 2} /\langle\rho\rangle$. For this reason Percus' relation (9) is also known as the "one-half rule" (see Fig 1). Finally, we mention that, as numerically proven in Ref.?, the presence of weak substrate disorder may induce superdiffusive SFD corresponding to $\alpha>1$.

- One-particle interaction. This type of interaction potential,

$$
U_{\text {int }}\left(x_{1}, \ldots, x_{N} ; t\right)=\int d X U(X ; t) \delta\left(X-x_{n}\right),
$$


was introduced to mimic the effects of an external field acting on one particle, alone ?. In this case, we agree to identify the tagged particle as the particle directly coupled to the potential. Popular examples are provided by optical or magnetic tweezers, which trap a single particle in a harmonic potential $U(X ; t) \equiv \omega^{2} X^{2} / 2$ with characteristic frequency $\omega$, while leaving the rest of the file unaffected. Moreover, if the tagged particle carries an electric charge, $q$, applying a constant external electric field, $E$, results in the one-particle potential $U(X ; t)=-F X$ with $F=q E$. In such a case and, more generally, for any constant field of force $F$, the ensuing drift of the tagged particle $\left\langle\left[x_{n}(t)-x_{n}(0)\right]\right\rangle_{F}$ fulfills the celebrated Einstein relation in the form ?, ?, ?, ????:

$$
\left\langle\left[x_{n}(t)-x_{n}(0)\right]\right\rangle_{F}=F \frac{\left\langle\left[x_{n}(t)-x_{n}(0)\right]^{2}\right\rangle}{2 k_{B} T} .
$$

Another kind of external interaction potential addressed in the recent literature, is the sinusoidal time-dependent drive $U(X ; t)=-A X \cos (\omega t)$ ?, ?. Under these conditions the Green-Kubo relation, for small enough $A$, still holds rigorously for the tagged particle,

$$
\left\langle v_{n}(\omega) v_{n}(-\omega)\right\rangle=4 \pi k_{B} T \frac{A}{\omega} \operatorname{Re}\left[\mu_{n}(\omega)\right],
$$

where $v \equiv \dot{x}$ and $\mu_{n}(\omega)$ is the complex mobility of the tagged particle defined via the identity $\left\langle v_{n}(t)\right\rangle=\operatorname{Re}\left[\mu_{n}(\omega) A e^{i \omega t}\right]$. However, the time modulation applied to the tagged particle affects, through the hard-core collisions, the dynamics of all remaining file constituents. In Refs. ?? the drift and velocity correlation functions of all untagged file particle were proven to fulfill generalized Kubo and Green-Kubo relations obtained as extensions, respectively, of Eqs.(12) and (13). Recently the same approach has been extended to a file system with distributed friction constants?.

- File particle-particles interaction. In this case no external field affects the file dynamics, while the particles are supposed to interact through some additional finite-range binary potential

$$
U_{\text {int }}\left(x_{1}, \ldots, x_{N} ; X, t\right)=\sum_{n, m=1} U_{\text {int }}\left(\left|x_{n}-x_{m}\right|\right)
$$

This kind of potential is particularly suited to describe real physical situations, where the single file particles are taken to be electrically or magnetically charged. As a matter of fact, several experiments have been carried out using particles with magnetic ?, electric dipole ???, or screened electrostatic pair interactions ??, ?, ?. These experimental studies provided strong evidence of the subdiffusive asymptotic behavior predicted in Eq.(11), despite an apparent slowing down in the case of screened electrostatic interactions ?. An exact analytical result was obtained for the SFD of tagged particles 
interacting through the quadratic potential

$$
U_{\text {int }}\left(\left|x_{n}-x_{m}\right|\right)=\langle\rho\rangle^{z} \frac{k_{z}}{4} A_{n, m}^{z}\left(x_{n}-x_{m}\right)^{2},
$$

where $k_{z}$ is a constant,

$$
A_{n, m}^{z}=\frac{\Gamma\left(|n-m|-\frac{z}{2}\right) \Gamma(z+1)}{\pi \Gamma\left(|n-m|+\frac{z}{2}+1\right)} \sin \left(\frac{z \pi}{2}\right),
$$

is a Toeplitz matrix ?, and $\Gamma(x)$ denotes a Gamma function with argument $x$. An explicit calculation shows that for $1<z<2$ the SFD formula of Eq.(1) must be replaced by $\left\langle\left[x_{n}(t)-x_{n}(0)\right]^{2}\right\rangle \propto t^{\frac{z-1}{z}}$ ?.

\section{Langevin equation for the tagged particle}

The question whether the tagged particle trajectory can be described by a bona fide Langevin equation upon integrating out all remaining file variables, was first addressed in Ref. ?. According to these authors the hard-core interactions of the tagged particle with its nearest neighbors was well reproduced by two phenomenological terms, to be regarded respectively as an intrinsic noise and a file damping term connected by a fluctuation-dissipation relation of the sort. The resulting phenomenological Langevin equation for the single file of Eqs.(2) and (4) with $U_{\mathrm{int}}=0$, reads

$$
\frac{d^{2} x_{n}(t)}{d t^{2}}+\gamma \frac{d x_{n}(t)}{d t}+2 \sqrt{\gamma k_{B} T}\langle\rho\rangle \frac{d^{1 / 2} x_{n}(t)}{d t^{1 / 2}}=\xi_{n}(t),
$$

where the Caputo fractional derivative is defined by ??

$$
\frac{d^{1 / 2} f(t)}{d t^{1 / 2}}=\frac{1}{\Gamma\left(\frac{1}{2}\right)} \int_{0}^{t} \frac{d f\left(t^{\prime}\right) / d t^{\prime}}{\left|t-t^{\prime}\right|^{\frac{1}{2}}} d t^{\prime}
$$

Such a fractional Langevin equation (FLE) has been validated by extensive numerical simulations ??. Moreover, it can be easily rewritten in the form of a generalized Langevin equation (GLE) ????

$$
\begin{aligned}
& \dot{x}_{n}(t)=v_{n}(t) \\
& \dot{v}_{n}(t)=-\int_{0}^{t} \kappa\left(t-t^{\prime}\right) v\left(t^{\prime}\right) d t^{\prime}+\xi_{n}(t)
\end{aligned}
$$

by introducing the phenomenological damping kernel $\kappa(t)=2 \gamma\left[\delta(t)+\langle\rho\rangle \sqrt{\frac{k_{B} T}{\gamma \pi t}}\right]$. The noise $\xi_{n}(t)$ is assumed to be Gaussian, zero-mean valued and related to the damping kernel by Kubo's generalized fluctuation-dissipation relation ?, i.e.

$$
\left\langle\xi_{n}(t) \xi_{n}\left(t^{\prime}\right)\right\rangle=k_{B} T \kappa\left(\left|t-t^{\prime}\right|\right) .
$$

Because of this property $\xi_{n}(t)$ is called fractional Gaussian noise. The Langevin description of Eqs. (17)-(20) closely reproduces all three diffusive regimes of the tagged particle and their time-scales: ballistic, diffusive and subdiffusive (see Fig 1). The success of this approach is largely due to the choice of the kernel $\kappa(t)$, where and 
ad hoc $\delta$ term was added to a subdiffusive long-lasting memory tail. As a matter of fact no rigorous derivation of the Langevin equations (17)-(19) has been put forward, so far. However, in the overdamped limit, the fractional terms of the Langevin equation (17) have been obtained thanks to a procedure called harmonization?. The resulting fractional Langevin equation for the tagged particle reads ?, ??

$$
2 \sqrt{\gamma k_{B} T}\langle\rho\rangle \frac{d^{1 / 2} x_{n}(t)}{d t^{1 / 2}}=\xi_{n}(t)
$$

where $\left\langle\xi_{n}(t) \xi_{n}\left(t^{\prime}\right)\right\rangle=2\langle\rho\rangle\left(k_{B} T\right)^{3 / 2} \sqrt{\frac{\gamma}{\pi\left|t-t^{\prime}\right|}}$. We hereby provide just a sketch of the derivation, addressing the interested reader to Ref.s ${ }^{\text {? }}$ for the specific details. The harmonization procedure aims at substituting the hard-core interaction (4) in Eq.(1) by its second order expansion around the maximum of the corresponding free energy, hence Eq.(2) transforms, in the overdamped limit, to

$$
\gamma \frac{d x_{n}(t)}{d t}=\langle\rho\rangle^{2} k_{B} T\left[x_{n+1}(t)+x_{n-1}(t)-2 x_{n}(t)\right]+\eta_{n}(t) .
$$

Passing to the continuum limit $x_{n}(t) \rightarrow x(n, t)$ and introducing the Fourier transform in space and time as $\tilde{x}(q, \omega)=\int_{-\infty}^{\infty} d n d t x(n, t) e^{-i(q n-\omega t)}$, the previous equation can be solved as

$$
\tilde{x}(q, \omega)=\frac{\tilde{\eta}(q, \omega)}{-i \omega \gamma+\langle\rho\rangle^{2} k_{B} T q^{2}} .
$$

Multiplying both sides by $2(-i \omega)^{1 / 2} \sqrt{k_{B} T \gamma}\langle\rho\rangle$ and performing an inverse Fourier transform in the $n$ domain we obtain

$$
2(-i \omega)^{1 / 2} \sqrt{k_{B} T \gamma}\langle\rho\rangle \tilde{x}(n, \omega)=\tilde{\xi}(n, \omega)
$$

where the fractional Gaussian noise is

$$
\tilde{\xi}(n, \omega)=\gamma \int_{-\infty}^{\infty} d n^{\prime} \tilde{\eta}\left(n^{\prime}, \omega\right) e^{-\sqrt{\frac{-i \omega \gamma}{\langle\rho\rangle^{2} k_{B} T}}\left|n-n^{\prime}\right|} .
$$

Inverting back in time, one recovers the Eq.(21). We stress that the very same equation can be immediately drawn from Eq 19 by time integration (using Laplace transform) and averaging over the initial velocities.

Besides its compact and elegant notation, the above formalism offers an additional remarkable advantage: all observables of practical interest can be calculated analytically. For instance, it becomes apparent that the subdiffusive law in Eq.(11) is closely related to persistent memory effects, which appear in the negative power-law tails of the velocity autocorrelation functions ???, ?, ??:

$$
\left\langle v_{n}(t) v_{n}\left(t^{\prime}\right)\right\rangle=-\sqrt{\frac{k_{B} T}{\gamma \pi}} \frac{\left|t-t^{\prime}\right|^{-3 / 2}}{4\langle\rho\rangle} .
$$

Physically, these long-time anticorrelations originate from the particle momentum exchange due to the hard-core collisions. By time integrating a velocity autocor- 
relation function, one obtains the corresponding position autocorrelation function, which in the long-time limit can be cast as??

$$
\left\langle\left[x_{n}(t)-x_{n}(0)\right]\left[x_{n}\left(t^{\prime}\right)-x_{n}(0)\right]\right\rangle=\sqrt{\frac{k_{B} T}{\gamma \pi\langle\rho\rangle^{2}}}\left[\sqrt{t}+\sqrt{t^{\prime}}-\sqrt{\left|t-t^{\prime}\right|}\right] .
$$

Now a stochastic process that exhibits persistent correlations, such those in Eq.(26), and noise autocorrelations, like $\left\langle\xi_{n}(t) \xi_{n}\left(t^{\prime}\right)\right\rangle \propto 1 / \sqrt{\left|t-t^{\prime}\right|}$, is said to perform a fractional Brownian motion (FBM) ?. Hence one could reasonably conclude that the tagged particle performs a fractional Brownian motion, which is true, except that in Mandelbrot's original definition of FBM no mention is made of any (generalized) fluctuation-dissipation relation. This is a delicate issue which goes beyond the purpose of the present article. The relation between FLE and FBM is addressed in Ref.?, while certain subtleties concerning the validity of the fluctuation-dissipation relation in the FBM are discussed in Ref.?

Yet, another worth mentioning aspect brought forth within the context of the FLE framework is the emergence of a universality class ??. As a matter of fact, the single file model belongs to a class of stochastic systems which includes several linearly interacting models in different area of physics, ranging from the one dimensional Edward-Wilkinson chain ??, to fluctuating interfaces ?, to Rouse polymers ?. In these many-body systems indeed, a tagged probe undergoes an anoumalous diffusion whose asymptotic behaviour is governed by the Eq.(21). Most importantly, the dynamics of the distance between the donor $(D)$ and the acceptor $(A)$ coordinates within a protein strain was shown to be reproduced, with an excellent degree of accuracy, by a FLE with an applied hookean force ?. In Ref.? it has been rigourously proven that the distance between two particles in single file systems, $\Delta_{n, n^{\prime}}(t)=x_{n}(t)-x_{n^{\prime}}(t)$, asymptotically recovers the FLE for the donor-acceptor distance (see also ?). Strictly speaking, the quantity $\Delta_{n, n^{\prime}}(t)=x_{n}(t)-x_{n^{\prime}}(t)$ behaves as a stochastic subdiffusive trajectory in an harmonic well, whose frequency is proportional to $\sqrt{n-n^{\prime}}$. Rephrasing this statement, two particles $n$ and $n^{\prime}$ are de facto connected by an entropic spring with stiffness equal to $\left|n-n^{\prime}\right|$.

We stress once more that, on jumping from the full file dynamics of Eq.(2) to the reduced GLE-FLE's (17)-(21) of the tagged particle, no systematic projection procedure was employed to eliminate the coordinates of the untagged file particles. Indeed, a controllable "adiabatic elimination" procedure of the fast variables, for instance, along the line of the Mori-Zwanzig formalism ????, cannot be carried out for a single file in the real space (as opposite to the Fourier space), where the time scales of all particle coordinates are identical. How are then the spatial file interactions of Eq.(2) accounted for in the phenomenological GLE formalism, Eqs. (17)-(21)? They partially translate into the time correlations contained in the definition of the fractional derivative, see Eq.(18), and in the spatio-temporal properties of the fractional Gaussian noise. Such a mechanism is apparent in the 
overdamped limit, where the autocorrelation of the fractional noise reads??

$$
\begin{aligned}
& \left\langle\xi_{n}(t) \xi_{n^{\prime}}\left(t^{\prime}\right)\right\rangle=\frac{\left(2 k_{B} T\right)^{3 / 2} \sqrt{\gamma}}{\pi} \int_{0}^{\infty} d \omega \frac{e^{-\frac{\mid n-n^{\prime}}{\langle\rho\rangle} \sqrt{\frac{\omega}{2 \gamma}}}}{\operatorname{los}^{\omega}} \\
& \left.\cos \left|t-t^{\prime}\right|\right)\left[\cos \left(\frac{\left|n-n^{\prime}\right|}{\langle\rho\rangle} \sqrt{\frac{\omega}{2 \gamma}}\right)+\sin \left(\frac{\left|n-n^{\prime}\right|}{\langle\rho\rangle} \sqrt{\frac{\omega}{2 \gamma}}\right)\right] .
\end{aligned}
$$

Note that for $n=n^{\prime}$ this expression of the noise autocorrelation coincides with Eq.(20). By making use of this property when taking the time integration of the FLE (21), one can easily calculate the long-time behavior of some particle-particle correlation functions, such as $\left\langle\left[x_{n}(t)-x_{n}(0)\right]\left[x_{n^{\prime}}\left(t^{\prime}\right)-x_{n^{\prime}}(0)\right]\right\rangle$ ?? or $\left\langle\left[x_{n}(t)-x_{n}(0)\right] v_{n^{\prime}}\left(t^{\prime}\right)\right\rangle$ ?

We now discuss how Eqs.(17)-(21) are modified by the presence of the interacting potential $U_{\text {int }}$.

- File interaction with a substrate. In this case no GLE for a tagged particle was ever proposed, not even in the overdamped limit. In particular, the question whether the harmonization technique can be extended to handle the case of a single file interacting with an underlying substrate, is still unanswered.

- One-particle interaction. When the external perturbation acts upon the tagged particle alone, an analytical derivation of the GLE for all file particles is still doable, at least in the overdamped limit. However, the resulting FLE will be different depending on whether one considers the tagged or untagged particles. In Ref.? the FLE of the tagged particle was derived for the case of a constant force. A general formulation valid for any kind of one-particle potentials was obtained in Ref.?. For the tagged particle the reduced FLE turned out to be

$$
2 \sqrt{\gamma k_{B} T}\langle\rho\rangle \frac{d^{1 / 2} x_{n}(t)}{d t^{1 / 2}}=-\frac{\partial}{\partial x_{n}} U\left(x_{n}, t\right)+\xi_{n}(t),
$$

where $U\left(x_{n}, t\right)=\int d X U(X ; t) \delta\left(X-x_{n}\right)$, like in Eq.(11). More remarkably, the FLE of an untagged particle of label $n^{\prime}$,

$$
\begin{aligned}
& 2 \sqrt{\gamma k_{B} T}\langle\rho\rangle \frac{d^{1 / 2} x_{n^{\prime}(t)}}{d t^{1 / 2}}= \\
& -\frac{\partial}{\partial x_{n}} \int_{-\infty}^{t} d t^{\prime} U\left(x_{n}, t^{\prime}\right) \Theta\left(\left|n-n^{\prime}\right|, t-t^{\prime}\right)+\xi_{n^{\prime}}(t),
\end{aligned}
$$

was shown to depend on the force propagator $\Theta$, i.e. the space-time Green's function which is carrying the external perturbation, while exerted on particle $n$ at time $t^{\prime}$, to the particle $n^{\prime}$ at time $t^{?}$. Indeed, although applied to the sole tagged particle, $n$, the perturbation propagates through the file via the hard-core collisions, until it finally reaches the untagged particle, $n^{\prime}$. The expression of $\Theta$ was obtained in terms of its time Fourier transform, that is

$$
\Theta\left(\left|n-n^{\prime}\right|, \omega\right)=\sqrt{\frac{\langle\rho\rangle}{\gamma}}\left(k_{B} T\right)^{1 / 4} e^{-\frac{\left|n-n^{\prime}\right|}{\langle\rho\rangle} \sqrt{\frac{-i \omega}{\gamma}}}
$$


We remark that the noise appearing in Eqs.(28) and (29) satisfies the same properties as the noise in Eq.(21).

- File particle-particle interaction. In the presence of binary interactions between nearest-neighbors, a tagged-particle FLE has been rigorously derived only for the quadratic interaction of Eq.(15) ?. In the long-time limit, the relevant FLE for $1<z<2$ boils down to

$$
z\langle\rho\rangle \sin \left(\frac{\pi}{z}\right) k_{z}^{1 / z} \gamma^{\frac{z-1}{z}} \frac{d^{\frac{z-1}{z}} x_{n}(t)}{d t^{\frac{z-1}{z}}}=\xi_{n}(t),
$$

where the fractional Gaussian noise satisfies the generalized fluctuationdissipation relation

$$
\left\langle\xi_{n}(t) \xi_{n}\left(t^{\prime}\right)\right\rangle=\frac{k_{B} T z\langle\rho\rangle \sin \left(\frac{\pi}{z}\right) k_{z}^{1 / z} \gamma^{\frac{z-1}{z}}}{\Gamma(1 / z)\left|t-t^{\prime}\right|^{\frac{z-1}{z}}} .
$$

\section{From the diffusion-noise equation to the fractional Langevin equation}

We address now the single file dynamics from a different viewpoint. Let us start from the Langevin equations (2) of a noninteracting single file with $U_{\text {int }}=0$; in Eq.(4) only the hard-core collision term, $U_{H C}$, is retained. We then concentrate on the time evolution of the particle density rather than on the particle dynamics. To do so, let us divide the ring in $M$ bins of length $\Delta X$ and define the coarse grained particle density

$$
\rho_{i}(t)=\frac{1}{\Delta X} \sum_{n=1}^{N} \vartheta\left[x_{n}(t)-(i-1) \Delta X\right] \vartheta\left[i \Delta X-x_{n}(t)\right],
$$

where $i$ is the bin label, $\vartheta$ denotes the Heaviside step function, and $\Delta X=L / M$. As we are interested in the thermodynamic limit, where the file equilibrium is characterized by the constant density $\langle\rho\rangle=\lim _{N \rightarrow \infty, L \rightarrow \infty} N / L$, the bin size can be taken arbitrarily small, $\Delta X \rightarrow 0$. Note that by virtue of the $\vartheta$ definition, $\vartheta[X]=\int_{-\infty}^{X} d s \delta(s)$, and the approximation $\int_{(i-1) \Delta X}^{i \Delta X} d s \delta\left(s-x_{n}(t)\right) \simeq \delta\left((i-1 / 2) \Delta X-x_{n}(t)\right) \Delta X$, the standard file density,

$$
\rho_{i}(t) \rightarrow \rho(X, t)=\sum_{n=1}^{N} \delta\left(X-x_{n}(t)\right),
$$

can be immediately recovered.

Describing the single file in terms of the coarse grained density surely implies a loss of information. Nevertheless, one can study the stochastic behavior of the bin density, $\rho_{i}(t)$, and try to relate its fluctuations to the tagged particle dynamics. To this purpose, we first notice that, being a local property of the file, the density definition in Eq. (32) does not depend on the particle relabeling upon collisions; differently stated, the coarse grained density of a single file does not change if we 
replace the non-passing file particles with noninteracting Brownian particles ?. We recall that in the absence of hard-core collisions the time evolution of the density profile is governed by the continuity equation?

$$
\frac{\partial}{\partial t} \delta \rho(X, t)=-\frac{\partial}{\partial X} J(X, t)
$$

where the density function has been separated into a noise average, $\langle\rho(X, t)\rangle$, and a fluctuating part, $\delta \rho(X, t)$, that is, $\rho(X, t)=\langle\rho(X, t)\rangle+\delta \rho(X, t)$. The density current appearing on the r.h.s. of Eq.(34) also evolves according to a stochastic equation,

$$
J(X, t)=-D \frac{\partial}{\partial X} \delta \rho(X, t)+\zeta(X, t),
$$

with a noise term, $\zeta(X, t)$, which satisfies the following identities

$$
\begin{aligned}
& \zeta(L, t)=\zeta(0, t), \\
& \langle\zeta(X, t)\rangle=0, \\
& \left\langle\zeta(X, t) \zeta\left(X^{\prime}, t^{\prime}\right)\right\rangle=2 D \delta\left(X-X^{\prime}\right) \delta\left(t-t^{\prime}\right)\langle\rho(X, t)\rangle .
\end{aligned}
$$

The periodic boundary condition formulated in the first identity is required to ensure the conservation of the particle number in the segment (or ring) $[0, L]$ (conserved noise $)$ ? The function $\langle\rho(X, t)\rangle$ encodes the spatio-temporal evolution of the noise-averaged density for given initial conditions, $\rho(X, 0)$. In particular, a uniform particle distribution at time $t$ corresponds to setting $\langle\rho(X, t)\rangle \equiv\langle\rho\rangle$. The set of Eqs.(34) and (35) is generally referred to as the diffusion-noise equation?. We also recall that Eq.(34) can be seen as the linear approximation of the Dean-Kawasaki equation ?, which constitutes the exact evolution equation for the microscopic density of a collection of interacting Brownian particles under overdamped dynamics ?.

The first authors who related the coarse grained density fluctuations to the mean square displacement of the tagged particles, were Alexander and Pincus ?. Thirty years later, another approach, still based on the density evolution equation (34), was proposed to obtain the same results, but under different approximations? We hereby review both approaches and show that they are indeed complementary. Before proceeding further, however, we mention two papers recently appeared on the connection between tagged particle dynamics and a liquid-theory approach at the mesoscopic scale, i.e. a Lagrangian formulation for the file density evolution ??. However it must be stressed that the above formalism holds for a system of interacting particles, while the present approach exploits the connection of an assembly of non-interacting Brownian particles and single file system.

On introducing the space Fourier transform of $\rho(X, t), \tilde{\rho}(Q, t)=$ $\int_{-\infty}^{\infty} d X e^{-i Q X} \rho(X, t)$, we rewrite Eq.(33) as

$$
\rho(X, t)=\int_{-\infty}^{\infty} d Q \sum_{n=1}^{N} e^{-i Q\left[x_{n}(t)-X\right]}
$$


which, in turn, yields

$$
\tilde{\rho}(Q, t)=\sum_{n=1}^{N} e^{-i Q x_{n}(t)} .
$$

Now, we write the trajectory of a tagged (non-passing) file particle as $x_{n}(t)=$ $\frac{n}{\langle\rho\rangle}+\delta x_{n}(t)$, upon assuming that the motion of the $n$-th particle takes place around its equilibrium position, $\left\langle x_{n}(t)\right\rangle=\frac{n}{\langle\rho\rangle}$. Accordingly,

$$
\tilde{\rho}(Q, t) \simeq \sum_{n=1}^{N} e^{-i Q \frac{n}{\langle\rho\rangle}}-i Q \sum_{n=1}^{N} \delta x_{n}(t) e^{-i Q \frac{n}{\langle\rho\rangle}},
$$

where deviations from the average particle position have been treated as perturbatively small, $\delta x_{n}(t) \simeq 0\left(\delta x_{n}(t) \ll Q^{-1}\right)$. The first term on the r.h.s. of Eq.(39) is the inverse Fourier transform of $\langle\rho\rangle$; hence,

$$
\delta \tilde{\rho}(Q, t) \simeq-i Q \sum_{n=1}^{N} \delta x_{n}(t) e^{-i Q \frac{n}{\langle\rho\rangle}} .
$$

On introducing the discrete Fourier transform of the particle trajectory, $\tilde{x}_{k}(t)=$ $\sum_{n=1}^{N} x_{n}(t) e^{-i \frac{2 \pi k}{N} n}$ (with $k$ integer wavenumbers $k=0, \pm 1, \pm 2, \ldots$ ), simple algebraic passages lead to the Alexander and Pincus relation connecting density and particle fluctuations, namely,

$$
\delta \tilde{\rho}(Q, t) \simeq-\left.i Q \delta \tilde{x}_{k}(t)\right|_{k=\frac{N Q}{2 \pi\langle\rho\rangle}} .
$$

To obtain the mean square displacement of the tagged particle under stationary conditions, it suffices now to note that $\left\langle\left[x_{n}(t)-x_{n}(0)\right]^{2}\right\rangle=2\left[\left\langle\delta x_{n}^{2}(0)\right\rangle-\left\langle\delta x_{n}(t) \delta x_{n}(0)\right\rangle\right]$. Moreover, the autocorrelation function $\left\langle\delta x_{n}\left(t_{1}\right) \delta x_{n}\left(t_{2}\right)\right\rangle$ can be readily calculated by means of Eq.(41). Such a task is greatly simplified in the continuum space limit, $x_{n}(t) \rightarrow x(n, t)$ and $\tilde{x}(q, t)=\int_{-\infty}^{\infty} d n x(n, t) e^{-i q n}$, with $q=\frac{2 \pi k}{N}$. On inverting Eq. (41), one eventually obtains the identity

$$
\begin{aligned}
& \left\langle\delta \tilde{x}\left(q_{1}, t_{1}\right) \delta \tilde{x}\left(q_{2}, t_{2}\right)\right\rangle \\
\simeq & -\left.\frac{\left\langle\delta \tilde{\rho}\left(Q_{1}, t_{1}\right) \delta \tilde{\rho}\left(Q_{2}, t_{2}\right)\right\rangle}{Q_{1} Q_{2}}\right|_{Q_{1}=q_{1}\langle\rho\rangle, Q_{2}=q_{2}\langle\rho\rangle},
\end{aligned}
$$

whose r.h.s. can be evaluated in the framework of the diffusion-noise equation, Eqs.(34) and (35), by making explicit use of the noise properties in Eq.(36). The final result is the autocorrelation function of the particle fluctuations?

$$
\left\langle\delta x\left(n, t_{1}\right) \delta x\left(n, t_{2}\right)\right\rangle \simeq \int_{0}^{\infty} \frac{d q_{1}}{\pi} \frac{e^{-D\langle\rho\rangle^{2} q_{1}^{2}\left|t_{1}-t_{2}\right|}}{\langle\rho\rangle^{2} q_{1}^{2}} .
$$


The asymptotic anomalous diffusion law of Eq.(11) can be easily recovered by rewriting Eq.(43) as

$$
\left\langle[x(n, t)-x(n, 0)]^{2}\right\rangle \simeq \frac{2}{\langle\rho\rangle^{2}} \int_{0}^{\infty} \frac{d q_{1}}{\pi} \frac{1-e^{-D\langle\rho\rangle^{2} q_{1}^{2} t}}{q_{1}^{2}},
$$

and explicitly calculating the integral there.

The alternative approach of Ref.? hinges on an explicit relation connecting particle fluctuations and current density. Such a relation can be derived immediately within the Alexander's and Pincus' former derivation. Indeed, by combining Eq.(41) and the Fourier transform of Eq.(34) in the $q$ domain, one has

$$
\left.\delta \tilde{x}(q, t) \simeq \int_{0}^{t} d t^{\prime} \tilde{J}\left(Q, t^{\prime}\right)\right|_{Q=q\langle\rho\rangle} .
$$

By taking the inverse Fourier transform in the coarse grained $x$ domain ( $n$ domain), it is obtained the approximate relation?

$$
\frac{d}{d t} \delta x(n, t) \simeq \frac{J\left(\frac{n}{\langle\rho\rangle}, t\right)}{\langle\rho\rangle},
$$

from which the integral expression (44) and the SFD law (1) follow suit. Although slightly different, Taloni and Lomholt approach provides additional insight into the single file dynamics. Indeed, their starting point was the coarse graining integral identity,

$$
\int_{x(n, t)}^{x\left(n^{\prime}, t\right)} d X \rho(X, t)=n^{\prime}-n
$$

which is equivalent, upon time derivation of both sides, to the spatio-temporal differential relation,

$$
\begin{aligned}
& \frac{d x(n, t)}{d t} \rho(x(n, t), t)-\frac{d x\left(n^{\prime}, t\right)}{d t} \rho\left(x\left(n^{\prime}, t\right), t\right) \\
= & -\int_{x(n, t)}^{x\left(n^{\prime}, t\right)} d X \frac{d}{d t} \rho(X, t),
\end{aligned}
$$

and, in view of the continuity equation (34), to the much simpler equality,

$$
\frac{d x(n, t)}{d t} \rho(x(n, t), t)=J(x(n, t), t),
$$

which holds for any $n$ and $n^{\prime}$. In a consistent coarse graining formalism, Eq.(46) is thus recovered by imposing $\rho(x(n, t), t) \simeq\langle\rho\rangle$ and $J(x(n, t), t) \simeq J\left(\frac{n}{\langle\rho\rangle}, t\right)$.

We take a step forward by writing the time Fourier transform of Eq. (45) as

$$
\left.\delta \tilde{x}(q, \omega) \simeq \frac{\tilde{\zeta}(Q, \omega)}{D Q^{2}-i \omega}\right|_{Q=q\langle\rho\rangle},
$$


where we again made use of Eqs. (34) and (35) and defined $\tilde{x}(q, \omega)=$ $\int_{-\infty}^{\infty} d t \tilde{x}(q, t) e^{-i \omega t}$. The corresponding inverse Fourier transform in the $n$-domain is

$$
\delta \tilde{x}(n, \omega) \simeq \int_{-\infty}^{\infty} d X \tilde{\zeta}(X, \omega) \frac{e^{-\left|\frac{n}{\langle\rho\rangle}-X\right| \sqrt{\frac{-i \omega}{D}}}}{2 \sqrt{-i \omega D}}
$$

or, equivalently,

$$
2 \sqrt{k_{B} T \gamma} \sqrt{-i \omega} \delta \tilde{x}(n, \omega) \simeq \int_{-\infty}^{\infty} d X \tilde{\zeta}(X, \omega) \gamma e^{-\left|\frac{n}{\langle\rho\rangle}-X\right| \sqrt{\frac{-i \omega}{D}}} .
$$

On further Fourier transforming back to the time domain, they finally recovered the FLE (21) with fractional time derivative $\frac{d^{1 / 2}}{d t^{1 / 2}} \delta x(n, t)=\int_{-\infty}^{\infty} \frac{d \omega}{2 \pi} \sqrt{-i \omega} \delta \tilde{x}(n, \omega) e^{i \omega t}$ ?

and fractional Gaussian noise $\xi(n, t)=\int_{-\infty}^{\infty} \frac{d \omega}{2 \pi} \int_{-\infty}^{\infty} d X \tilde{\zeta}(X, \omega) \gamma e^{-\left|\frac{n}{\langle\rho\rangle}-X\right| \sqrt{\frac{-i \omega}{D}}+i \omega t}$ (see Eq.(25) ?,?.

Finally, we notice that the harmonization technique could be extended to the interaction with a substrate within he diffusion-noise approach, following the Dean's oute traced for a system of interacting Brownian particles.

\section{Conclusions}

In this paper we reviewed select advances in the field of single file diffusion. Our attention focused mostly on the effective stochastic equation that governs the motion of a single file particle, the so-called tagged particle, when the time evolution of the remaining file particles is ignored or not accessible. Such an equation allows the direct analytical calculation of all correlation functions and, therefore, of all relevant physical observables. For instance, dynamical structure factors and file transport properties ultimately depend on the asymptotic diffusion law of the tagged particle. Moreover, the generalized Langevin framework encompasses the presence of external perturbations in a very intuitive and analytically tractable form. Changing perspective, we have shown how the fluctuations of the density profile along the substrate are strictly related to the random motion of tagged particle. This relation, obtained through two different complementary approaches, sheds light on the intimate connection existing between tagged particle and density fluctuations and, equivalently, between fractional Langevin equation and diffusion-noise equation formalism. This constitutes a truly powerful theoretical tool, allowing an observer to infer the diffusion properties of a single particle from the spatio-temporal sampling of the overall file density. 
14 Alessandro

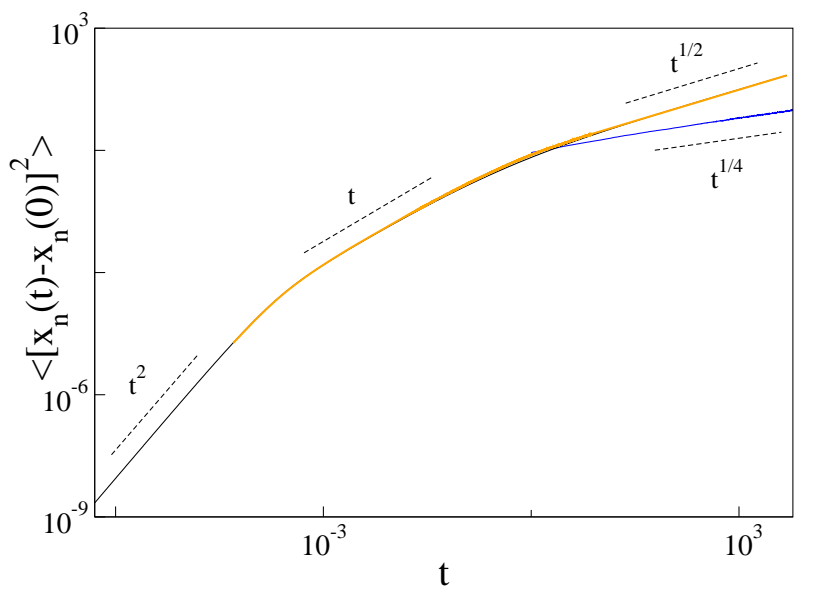

Fig. 1. Tagged-particle mean-square displacement curve (solid orange) showing the three characteristic diffusive regimes: ballistic, diffusive, and subdiffusive. The corresponding theoretical prediction (solid black curve) was obtained by numerically integrating Eq. (17). Integration parameters are $\langle\rho\rangle=0.1, k_{B} T=1.0$, and $\gamma=0.1$. The subdiffusive behavior of a tagged particle in a single file of subdiffusing particles, $\left\langle\left[x_{\text {free }}(t)-x_{\text {free }}(0)\right]^{2}\right\rangle \propto t^{\alpha}$ with $\alpha=0.5$, see Eq. (9), is shown as a test of the one-half rule. 